\title{
The Contribution of GGP Superconducting Gravimeters to GGOS
}

\author{
David Crossley \\ Department of Earth and Atmospheric Sciences, Saint Louis University, 3642 Lindell Blvd. St. Louis, MO 63108, \\ USA, crossley@eas.slu.edu \\ Jacques Hinderer \\ EOST/Institute de Physique du Globe, 5 Rene Descartes, 67084 Strasbourg Cedex France
}

\begin{abstract}
.
The network of more than 24 superconducting gravimeters (SGs) of the Global Geodynamics Project (GGP) is available as a set of reference stations for studies related to time-varying gravimetry. The inherent stability of the SG allows it to detect signals from a sampling time of $1 \mathrm{~s}$ up to periods of several years with a time-domain accuracy of 0.1 $\mu \mathrm{Gal}$ or better. SGs within the GGP network comprise a valuable set of stations for geodetic and geophysical studies that involve Earth's surface gravity field. Experience has shown that SGs can be calibrated to an accuracy of $0.01-$ $0.1 \%$, and that most instruments have a low, but well-modeled, drift of a few $\mu$ Gal /yr. For most purposes except the determination of an absolute gravity reference level, the SG is the best observation-style instrument we have today. SG data is now freely available, much of it going back to the early 1990's, from the GGP database at ICET (International Centre of Earth Tides, in Brussels, Belgium) and GFZ (Potsdam, Germany). Frequently it is combined with other datasets such as atmospheric pressure and hydrology for studies of ground deformation and tectonics. One of the most interesting new ideas within GGOS (Global Geodetic Observing System) is the determination of the geocenter using a combination of satellite and ground-based gravimetry. The GGP network can provide a unique contribution through continuous data at the stations where absolute gravimeters (AGs) will be deployed. The combination of the two instruments is necessary to ensure that AG measurements are referencing the mean station gravity and not short-term gravity perturbations due for example to hydrology or meteorology. Another promising application is the use of SG sub-networks in Europe and Asia to validate time-varying satellite gravity observations (GRACE, GOCE).
\end{abstract}

Keywords. Superconducting, gravimetry, GGP, GRACE

\section{Introduction}

The GGOS Reference Document (Plag, H.-P. and Pearlmanm M., 2007) is a draft document and hence cannot be cited directly, but it is the basis of the issues discussed in this article. The reference document describes, in great detail, the rationale for a future ideal network of high quality observing site for a number of important issues in geoscience such as mass changes at the Earth's surface due to climate change. These observing sites are complemented by an array of satellites that measure the Earth's gravity field (e.g. gravity recovery and climate experiment, GRACE) and observe the terrestrial reference frame (e.g. satellite laser ranging, SLR). GGOS, as a full component of the IAG, is an umbrella for the many geophysical and geodetic systems that are either in place, or need to be developed in the next decade (the target date is 2020). GGP is identified within GGOS as one of the groups responsible for monitoring the relative changes in the gravity field. GGP currently has about 25 operating

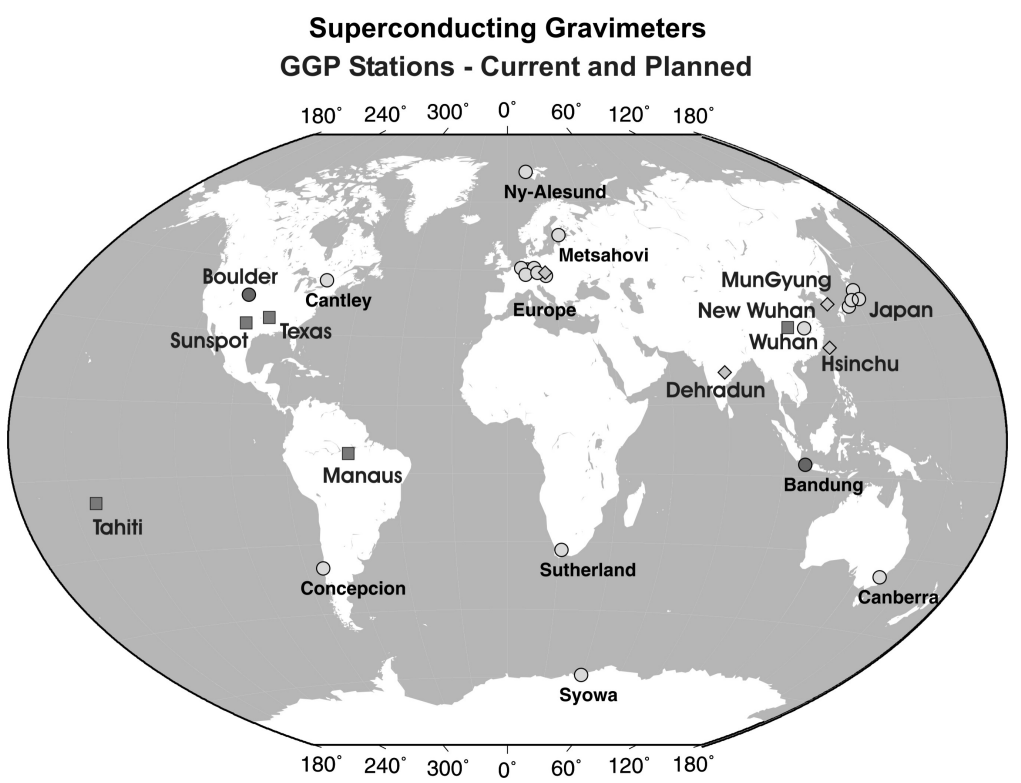

Figure 1. Distribution of SG stations: light circles are operational, dark circles are stopped, diamonds are new installations, and squares are planned. 
superconducting gravimeters (SGs), a few of which will probably be replaced or relocated within the next few years (Figure 1). If all existing and planned stations remain operational, there could be as many as 30 GGP stations available to GGOS by about 2010. Here we consider to what use these stations can be put for the GGOS requirements to be realized by 2020 .

\section{GGP Operations}

GGP has been operating officially for just 10 years (Crossley et al., 1999; Crossley and Hinderer, 2007), but has data archived back to the 1980's for some instruments. This data base is the primary achievement of GGP, and is freely available 1 year after collection by logging on to http://ggp.gfz-potsdam.de. From the 1 minute uncorrected data it is relatively simple to make obvious 'corrections' for missing data, or to make gaps for bad data. This is done on a regular basis by ICET personal to provide ' 1 minute corrected' data that is particularly suitable for tidal analysis. The 1 minute data is very good for short period studies such as tides (up to diurnal periods), and studying atmospheric effects in gravity and connections with hydrology. The difficulty is to extract accurate long-timescale field variations where the inevitable drift (however small) and disturbances and offsets need to be carefully determined.

The latter is an aspect where GGP experience can contribute to GGOS (Global Geodetic Observing System). GGP groups have now become expert on the treatment of data and searching for small signals and interpreting many of the field variations. Such information is circulated in GGP Newsletters and the proceedings of GGP Workshops, as described in Crossley and Hinderer (2007). Many of the personnel doing work with SGs will inevitably be involved in GGOS measurements, and thus be able to draw on their GGP experience for GGOS projects.

There are new aspects of GGP operations that can be exploited for GGOS benefit. The first is to add GPS data from GGP sites that are perhaps not otherwise available. The second is to provide access to absolute gravimeter (AG) measurements at SG sites, those measurement having been made with respect to the calibration of the SGs, or establishing the drift level of the SG (frequently both). These are tasks that GGP has long discussed (particularly the archiving of AG data), and if they deemed important to GGOS, this gives us useful incentive.

\subsection{SGs at Fundamental Stations}

GGOS defines a fundamental station as a site where at least 3 independent space-geodetic techniques are co-located. Additionally, as stated in the Reference Document, there 'should be' AG and SG observations and tide gauges 'where possible'. GGOS lists 25 such stations in current operation.

Only a few SGs are located at current fiducial geodetic sites, i.e. those with multiple large instruments such as VLBI, SLR, and DORIS, for geodetic work (Table 1). These sites are: Medicina, Metsahovi, Canberra, Ny-Alesund, Syowa, Tigo-Concepcion, and Wettzell. All GGP sites have GPS and meteorological equipment and many have some way to assess local hydrological variables such as groundwater. There is an increasing use of soil moisture meters within GGP, based on the need for such measurements in the demonstrated success of global hydrology models such as GLDAS (Rodell et al., 2004). We note also that many of the GGP sites have other geodynamical equipment such as seismometers and tiltmeters.

A subset of new stations in Table 1 is being planned by individual groups. In the future, station Sutherland that is now co-located with a number of optical telescopes - may become a fully operational GGOS site

Table 1. GGP sites co-located with two or more space geodetic techniques.

\begin{tabular}{|c|c|c|c|c|c|c|c|c|}
\hline Code & Location & Country & Latitude & Longitude & GPS $^{*}$ & VLBI & SLR & OTHER \\
\hline CA & Cantley & Canada & 45.5850 & 284.1929 & $y$ & & & satellite \\
\hline CB & Canberra & Australia & -35.3206 & 149.0077 & $y$ & & $y$ & DORIS \\
\hline ES & Esashi & Japan & 39.1511 & 141.3318 & $y$ & & & tiltmeters \\
\hline MC & Medicina & Italy & 44.5219 & 11.6450 & $y$ & y & & \\
\hline ME & Metsahovi & \begin{tabular}{|l|} 
Finland \\
\end{tabular} & 60.2172 & 24.3958 & $y$ & $y$ & $y$ & DORIS \\
\hline NY & Ny-Alesund & Norway & 78.9306 & 11.8672 & $y$ & $y$ & & DORIS \\
\hline SU & Sutherland & S. Africa & -32.3814 & 20.8109 & $y$ & & & optical \\
\hline SY & Syowa & Antarctica & -69.0067 & 39.5857 & $y$ & $y$ & & DORIS \\
\hline TC & Concepcion & $\begin{array}{l}\text { Chile } \\
\end{array}$ & -36.8437 & 286.9745 & $y$ & $y$ & $y$ & \\
\hline WU & Wuhan & China & 30.5159 & 114.4898 & $y$ & & $y$ & DORIS \\
\hline WE & Wettzell & Germany & 49.1440 & 12.8780 & $y$ & $y$ & $y$ & LLR, DORIS \\
\hline & & & & & & & & laser-gyro \\
\hline na & Sunspot NM & USA & 32.7660 & -105.8200 & $y$ & & & LLR, DORIS \\
\hline na & Tahiti & France & -17.5769 & -149.6063 & $y$ & $y$ & $\mathrm{y}$ & DORIS \\
\hline
\end{tabular}
with SLR, LLR and possibly VLBI. A probable future GGP location will be Tahiti that is now a geodetic fiducial station. To date there has been no master plan for the development of the GGP network, and no declaration that fiducial stations need to include SGs. This is one of the items we hope that GGOS will be able to clarify in the near future.

Thus under the present planning, the proportion of all GGP sites that are fiducial stations will remain at about $30 \%$. The interesting, and by no means easy-to-answer, question to be addressed within the gravity and geodetic communities is to what extent does knowledge of time-variable gravity assist in the fundamental geodetic work of the fiducial stations? Continuous gravity variations (arising from local mass changes) are considered important by GGOS to improve the geodetic solutions, which is why an AG and SG are specified at each of the future GGOS fundamental stations. If the current GGOS plan to extend the number of fundamental stations to $30-40$ by 2020 is feasible, then we need to extend the GGP network to more of the future GGOS stations.

A question that arises with any observing system is its lifetime, defined through the performance of the instruments and the goals and resources of the institutions that support them. Within GGP the majority of the stations have continued recording for up to 10 years or more. The issue of the possible closure of ground stations, whether from changes in personnel or policies, has affected GGP operations, but not so far compromised the viability of the network. 
It would be a good idea if vulnerable ground stations that are essential to GGOS should be identified as early as possible so that appropriate countermeasures could be taken. GGP has been built from the goals of individual projects, but in future if GGP could have a global mission directly connected with GGOS, this may discourage established stations from fading away. Fiducial stations, or at least stations with multiple instruments, have a heavy investment, which is both an advantage and a disadvantage. Initial and continuing costs are substantial, and the investment demands continued support. On the other hand, the same investment and costs can be justified by a wider variety of projects, and such sites are probably less likely to lose instruments because they use resources more efficiently than isolated installations.

\section{GGOS Gravity Field Specifications}

\subsection{Resolution}

Of central importance to GGOS is the question of what resolution (i.e. what time and distance scales) is required of the gravity field in order to address the important scientific questions. We agree with the GGOS emphasis on hydrology at regional scales as the most problematic of the tasks facing accurate geodetic measurements, and that spacecraft tracking is the most demanding external goal. GGOS suggests that the surface gravity field should be available at length and time scales of $50 \mathrm{~km}$ and 10 days. This resolution is not currently available by any combination of techniques (satellite or ground). Satellite gravimetry has vastly superior spatial coverage, but it is difficult to extract useful information at wavelengths as short as $50 \mathrm{~km}$. GRACE can measure sub- $\mu$ Gal levels, but only at wavelengths of a $400-1000 \mathrm{~km}$. Traditional GRACE solutions have been limited to 1 month field updates, but some recent GRACE-derived solutions are able to generate models at the 10-day time resolution (http://bgi.cnes.fr:8110/geoid-variations/README.html), so the GGOS time specification appears realistic in this respect.

\subsection{Accuracy and Noise Levels}

The accuracy also is important, but it is not simply an instrumental problem. GGOS rightly frames the question in terms of the need to know the geoid to a 1 $\mathrm{mm}$ level with a stability of $0.1 \mathrm{~mm}$ in order to have a TRF that is an order of magnitude more precise than the phenomena to be monitored. SGs and AGs can do spot ground measurements of the gravity field itself at accuracies of about $0.1 \mu \mathrm{Gal}$ and $1 \mu \mathrm{Gal}$ respectively (the precision of the SG is much better, about 1-10 nGal, but this is difficult to verify observationally). Thus in SG-AG combination we can claim to be measuring the gravity field at accuracies that are typical (but not an order of magnitude below) ground noise levels at periods of seconds to years. The ambient noise levels are set by seismic energy release, ocean circulation, the atmosphere and different contributions to hydrology, all

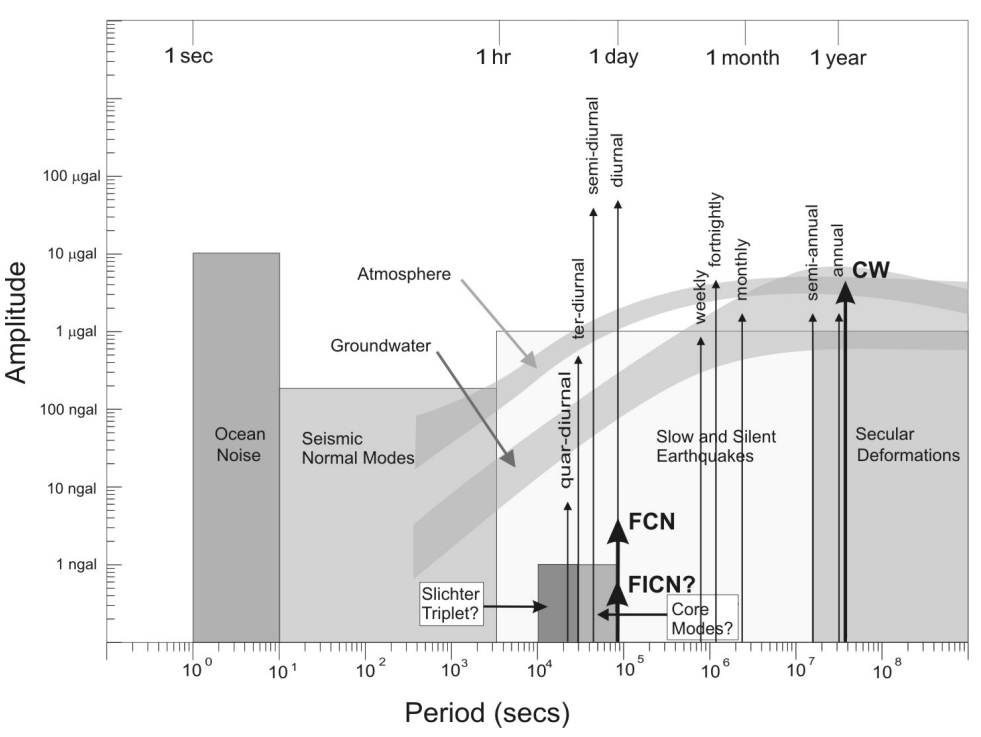

Figure 2. Amplitude of known effects on surface gravity. Some signals are at discrete periods (lines), and others are equivalent normalized amplitude spectra (atmosphere, groundwater) or are represented by blocks.

of which compete at about the $1 \mu \mathrm{Gal}$ level at a wide range of periods (Figure 2). In the seasonal and secular period ranges, tectonics competes mainly with hydrology and atmospheric effects at the several- $\mu$ Gal level.

We demonstrate these statements in Figure 3 that shows a 10-year series of residual data (1 day samples) from Strasbourg, both in its original form, and with a linear trend removed. The purpose is to show that continuous variations of gravity occur at all timescales at typical levels of 1-3 $\mu \mathrm{Gal}$ over periods of weeks with larger fluctuations of 5-10 $\mu \mathrm{Gal}$ at longer periods known to be caused by the annual hydrology cycle. The inset of 6 months of data (at $1 \mathrm{hr}$ samples) shows variations at the few $\mu$ Gal level that are typical of SG data at SG stations, but are not generally identified with any one cause. These high accuracy recording are point measurements, and are dependent on the gravimeter location with respect to soil moisture (see later). From a GGOS perspective, it would be very difficult to establish the gravity field at $50 \mathrm{~km}$ spatial resolution with this type of SG precision. 


\section{Examples of GGP Applications to GGOS}

We present some GGP results that are relevant to GGOS, primarily in the areas of combined AG-SG observations and hydrology, as the combination of instruments is best suited to long-term changes in the gravity field at seasonal periods and longer.

\subsection{AG Measurements at GGP Sites}

There are many examples (too many to be cited here) of the combined use of AG and SG measurements in the literature because almost all SGs are calibrated by an AG, often several times a year. The calibration aspect is required a few times after the SG is installed, but repeated calibrations have rarely yielded significant changes to the SG scale factor, as can be seen in Figure 4 (Amalvict and Hinderer, 2007). Their overall weighted mean value is $-79.14 \pm 0.30$, which is within $0.07 \%$ of the nominal value first measured and listed in the GGP headers for this station. It is interesting to note that the calibration is independent of whether individual drop means or set means are used. The optimum time to establish a calibration is about 5 days. The SG calibration also does not depend on which FG5 (the most common model of AG) is used for the calibration (Francis and Van Dam,

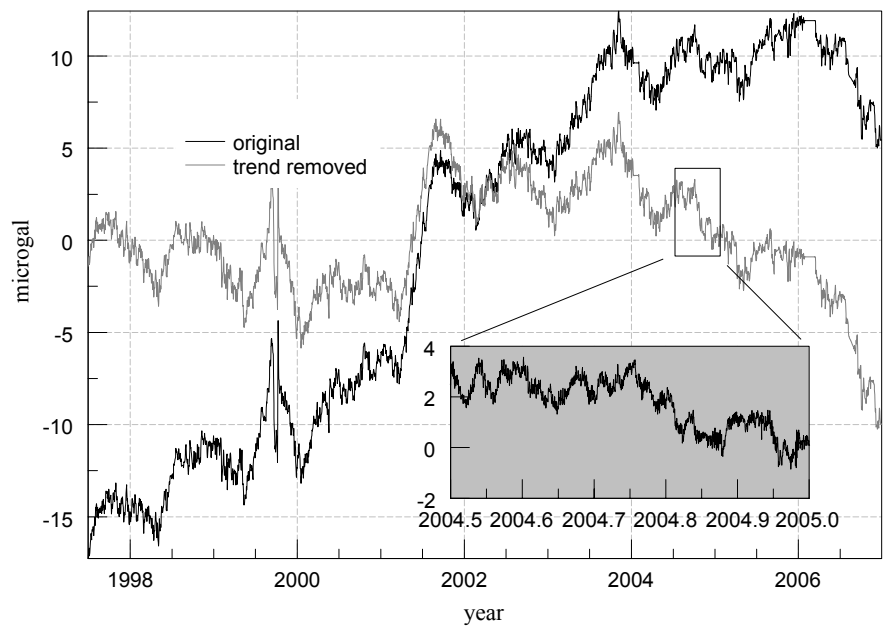

Figure 3. Residual SG data from Strasbourg showing original series (black), and after removal of linear trend (gray). The inset shows 6 months of the same data between July and December 2004. Tides, nominal pressure, and polar motion, have been removed, and the data cleaned of disturbances and offsets.

2002), and a host of experiments suggests that the calibration can be done successfully to $0.1 \%$ or better.

Other than calibration, repeated AG measurements serve instead two other important functions - first as a check on major offsets in an SG series of a non-instrumental nature, (e.g. power supply problems or disturbances during $\mathrm{He}$ refills) and as a check on instrument drift, and second, to verify that the $A G$ is functioning as expected. This latter issue may seem surprising, but in reality AGs are subject to occasional offsets and level problems that must be verified either by intercomparison campaigns (e.g. Francis et al., 2005) or using an SG (e.g. Imanishi et al., 2002).

An example of the latter is shown in Figure 5 in which we show at Strasbourg SG continuous gravity, hydrology from two different models (LaD and GLDAS) and several AG 'points' from 3 different FG5s. The SG series is the same as the slightly longer data set in Figure 3 (black curve). Note that part of the trend in Figure 3, and all of it in Figure 5 (the difference being SG drift), is real because the overall increase in gravity at Strasbourg is verified by AG measurements. All 3 series agree to first order, suggesting that

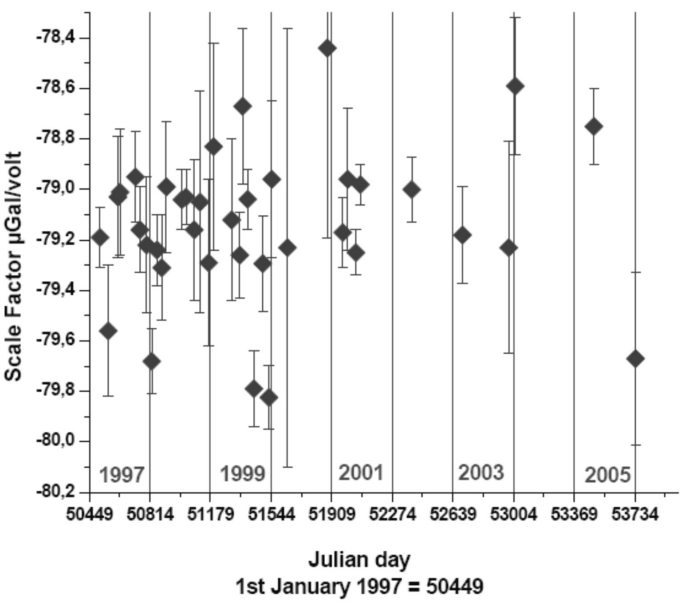

Figure 4. Scale factor for SG C026 in Strasbourg, calibrated by FG\#206 over 8 years. (Amalvict and Hinderer, 2007)

hydrology can explain most of the AG trend; the remainder is tectonically related. At the end of the series in 2007 (Figure 5) the gravity loses the upward trend, for reasons that are still being considered. We note the AG measurements sometimes deviate from the SG series for no apparent reason, i.e. when there are no obvious offsets or disturbances in the SG series and when the AG set values seem 'good'. Stray single measurements are expected, but note the cluster of AG values around day 53004 that are several $\mu \mathrm{Gal}$ high than the SG series.

We end this brief review of AG-SG combinations by referring to our German colleagues who reported in a series of papers (e.g. Wziontek et al., 2006; Wilmes et al., 2007) on the combination of 4 AGs and a dual-sphere SG in Bad Homburg, with strict processing and impartiality of the treatment of the results. Interestingly, they find that the SG calibration can be made successfully and consistently only when offsets of about 1-4 $\mu$ Gal are introduced into the AG series (after allowing for monument offsets and all other possible causes) over time spans of a year. We cannot pursue discussion on the precision of AGs, see the many other papers such as van Camp et al. (2005), but simply stress the advantages of an SG to check for potential problems. Early on, Okubo et al. (1997) pointed out that an SG can be used to verify AG measurements to $1-2 \mu \mathrm{Gal}$ (the anticipated and oft-quoted accuracy) under controlled conditions. When so 
checked, this allows the AG data to be usefully combined with tide gauge and GPS data to determine the cause of sea level changes, an issue of central importance to GGOS.

Nevertheless, it seems unavoidable to conclude that: (a) without frequent $\mathrm{AG}$ measurements at a site, questionable (i.e. not obvious) disturbances or offsets in the SG cannot be identified, and obviously no check on instrumental drift, and (b) without an SG (or a second AG) it is possible that unknown offsets may have crept into an AG determination. So SGs and AGs are complementary - they serve to check each other by entirely independent observations.

\subsection{Tectonic Uplift Using AGs}

One example of a comprehensive AG campaign has been described by Timmen et al. (2006). The proposed network is shown in Figure 6, consisting of many established stations and many others not yet occupied, particularly in the Eastern section, to complete the coverage. The campaign will establish 30 stations to be surveyed annually, with an estimated $3 \mu \mathrm{Gal}$ accuracy from a single determination. The authors strongly recommend parallel recording by two AGs where possible to improve the reliability of the network. Combined with GPS, such a network is a powerful tool for geodynamics, even though the effort needed to continuously deploy the 3-4 AGs involved is considerable. Only one of the stations is tied to an SG, at Metsahovi. The target here is to validate GRACE measurements that are at the $1 \mu \mathrm{Gal}$ accuracy at wavelengths of $1000 \mathrm{~km}$. Post-glacial uplift is associated with changes of about $0.6 \mathrm{~mm} \mathrm{yr}^{-1}$ in the geoid and $2 \mu \mathrm{Gal} \mathrm{yr}^{-1}$ changes in gravity, at the maximum amplitude.

Uplift projects inevitably raise the question of the gravity / height ratio. Wahr et al. (1995) showed that the traditional free air effect of $-0.31 \mu \mathrm{Gal} \mathrm{mm} \mathrm{m}^{-1}$ is modified by viscous vertical displacement, reducing the value to $-0.15 \mu \mathrm{Gal} \mathrm{mm}^{-1}$. A recent paper by de Linage et al. (2006) shows that the ratio is strongly modified by surface hydrology loading effects, with a global mean value of $-0.86 \mu \mathrm{Gal} \mathrm{mm}^{-1}$ determined using the LaD model (Milly and Shmakin, 2002). The ratio varies according to the loading and basin size. Naturally the intercomparison of GRACE and surface gravity involves also knowing the ellipsoidal height changes of the AG from GPS height observations. Fukuda et al. (2007) showed some interesting contradictory results on uplift in Antarctica between GRACE solutions (positive gravity trend) and ground AG measurements (negative), where they suggest that satellite data may be used to assess the regional hydrology, leaving the ground results to deal with the local hydrology and height variations. It is more usual that AG projects such as that outlined in Antarctica by Rogister et al. (2007) must proceed without SG support.

\subsection{Geocenter Variation by AG and GPS}

One of the projects identified by GGOS is to determine the geocenter position to the same accuracy as the geoid, i.e. $1.0 \mathrm{~mm}$ stable to $0.1 \mathrm{~mm}$. Typically this parameter is a solution from SLR and similar techniques (e.g. Wu et al., 2006) but it is also possible to use ground gravimetry at stations where AG and GPS data are available to tie the center of mass (CM) to the the origin of the TRF. The accurate estimation of the gravity / height ratio (Plag et al. 2007) again involves the small amplitude of the desired signal (secular gravity) to noise levels of similar size (hydrology, atmosphere), at long periods. The best way to improve the gravity part of the gravity/height ratio is the co-location of different gravity techniques (AS-SG) at a single station, or the use of close-packed arrays of instruments. Interestingly, 


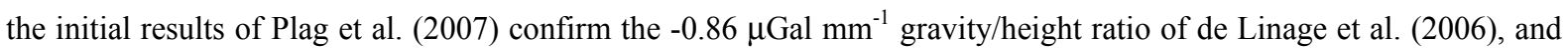
this leads to a shift in the Z-component of the $\mathrm{CM}$ of about $1.8 \mathrm{~mm} / \mathrm{yr}$, similar to that found by other methods.

\subsection{Practicality on AG-SG Combinations}

It is impractical to insist that an SG can be placed at every AG site, even for GGOS. Not every site is suitable for a continuous installation, particularly as SG measurements need to continue for some months or even years generally to be useful. It is reasonable, however, to suggest that as many SG stations as possible be included in GGOS projects that involve AG measurements, particularly as the latter are done regularly in any case. There are obvious advantages to GGOS if the GGP network can be expanded to more of the key AG sites where there is infrastructure to support an SG. For some of the GGOS projects (e.g. the ground-based estimation of the geocenter determination) it is not necessary to have the gravity / GPS measurements at space geodetic sites.

\section{Hydrology Effects}

There are now many studies of the gravity effect of soil moisture and groundwater using a variety of instruments. All of them confirm the complexity of finding a simple admittance between surface gravity and a variable such as groundwater.

\subsection{Gravity / Groundwater Admittance}

This relationship becomes especially complicated at sites where the gravimeter is not just close to the surface, but below it; for example in an underground bunker or the basement of a building. The problem was recognized in the analysis of SG data with the predictions of hydrological models such as LaD (Milly and Shmakin, 2002) and GLDAS (Rodell et al., 2004) in the studies of GGP stations in Europe (e.g. Crossley et al., 2006, Boy et al., 2006). Stations such as Strasbourg, Membach, and Moxa show a mixed hydrological signal that is more complicated than at Wettzell for example (Figure 7), where the correlation is much higher and a Bouguer slab approximation for groundwater storage is more reasonable (e.g. Crossley et al, 1998). Recent studies at these stations have been done by Longuevergne et al. (2007) for Strasbourg, Meurers et al. (2007) for Membach, and Kroner and Jahr (2006) for Moxa). They all show that detailed modeling can be used in explaining how the soil moisture and groundwater signal is divided both above
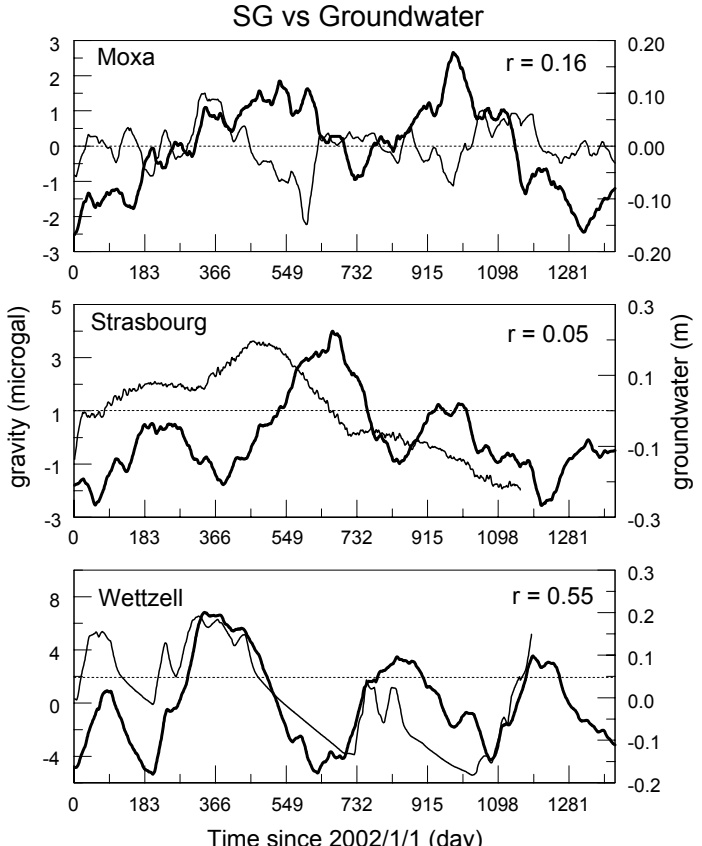

Figure 7. Correlation between groundwater level (light curve) and SG gravity (dark curve) at 3 stations in Europe. Wettzell is the only SG above ground.

and below the instrument. Only when a detailed model has been shown to work is it possible to consider the actual 'correction' of gravity data for hydrology (e.g. Meurers et al., 2007).

Longuevergne et al. (2007) showed the agreement at the Strasbourg GGP station between rainfall, soil moisture, and gravity changes at the 1-5 $\mathrm{GGal}$ level (Figure 8). Note the sudden reduction in gravity caused by soil moisture, here in a layer only a few m thick above the station. It is especially useful to see the AG measurements that are able to track the hydrology variations, and confirm the SG (and vice-versa). Without the SG, one might easily just ascribe the AG discrepancies to measurement errors.

Clearly the continuous SG sampling, (1-5 s for gravity and $1 \mathrm{~min}$ for hydrology), and the accuracy of $0.1 \mu \mathrm{Gal}$ or better is ideally suited to study these effects. Together with readily available rainfall data, the response of a particular site to meteorological forcing can be studied in detail. The problem becomes more complicated if one wants to assess the contribution of local hydrology to gravity, say in a $1 \mathrm{sq} \mathrm{km}$ area surrounding the instrument. This type of study out of practicality uses portable spring gravimeters due to their ease of set up and relatively modest costs (cf. SG, AG), but they have other problems associated with limited accuracy

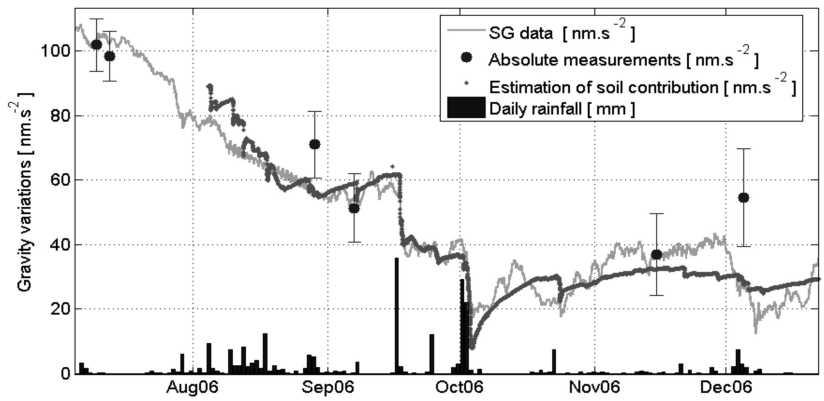

Figure 8. Contribution of soil moisture to gravity at an SG below ground level (Longuevergne et. al, 2007). and high drift. 
Smith et al. (2007) note the difficulties of measuring hydrology with the Scintrex CG-3M, whose nominal accuracy is only $5 \mu \mathrm{Gal}$. They review the strategies for making corrections to find the desired signal whose size is at the ground noise level and accuracy limit of the instrument. Similar studies reported by Dijksma et. al, (2006) using the same type of instrument located only $0.75 \mathrm{~m}$ below the surface, found gravity signals in the range $1-3 \mu$ Gal that would have been closer to $10 \mu \mathrm{Gal}$ if the instrument had been located at ground level, with all hydrology below the instrument.

A comprehensive study is currently underway in Moxa (Naujoks et al., 2006) using a set of 4 well-calibrated and maintained Lacoste and Romberg ET (spring) gravimeters to monitor gravity at intervals of several months at 390 stations (!). The SG is near the bottom of a valley with steep, vegetation-dominated, sides, and water flow is all around the gravimeter. In general the porosity and permeability of the overburden layers must be well known to do the modeling accurately. Their second paper (Naujoks et al., 2007) included detailed modeling and comparison with the ET network observations. They showed that $80 \%$ of the gravity variations could be modeled by hydrological changes within a $0.5 \times 0.5 \mathrm{~km}$ area around the SG. Further, the gravity variations were in the range $2-10 \mu \mathrm{Gal}$, above the estimated $1 \mu \mathrm{Gal}$ accuracy of the Lacoste \& Romberg ET (spring gravimeter) observations (after all corrections), and therefore sufficient to be compared with the models.

\section{Conclusions}

Our main conclusions for AG measurements are thus:

1. hydrological variations - primarily in soil moisture and groundwater, but also in ice mass changes for stations such as NY and SY - are normally the largest unmodeled effect on AG measurements,

2. the conversion of rainfall to gravity effect at a station rarely can be done with a simple admittance; it requires detailed modeling using a high accuracy gravimeter such as an SG, and

3. when AG measurements are require to detect geophysical effects at the limit of AG precision (1-2 $\mu \mathrm{Gal})$, especially by measurements that are spaced more than a few days apart, the use of a co-located SG will significantly enhance the quality of the detection of long-term changes.

In contributing to GGOS goals, we have seen that GGP can:

4. share experience in use of the GGP database with GGOS representatives (through Newsletters, GGP Workshops, etc.),

5. record and report on all GPS measurements at GGP stations - these are observed as height variations that contribute to gravity variations,

6. record and report all AG measurements made at the GGP sites - these would be benchmark measurements (one point with error bar and supplementary information),

7. participate in future campaigns to inter-compare AGs at a site, where there is an SG, and

8. join initiatives in geodesy or tectonics where the use of an SG would significantly improve the interpretation of measurements from other instruments.

\section{References}

Amalvict, M., Hinderer, J., and Rozsa, S., 2006. Crustal vertical motion along a profile crossing the Rhine graben from the Vosges to the Black Forest Mountains: Results from absolute gravity, GPS and leveling observations, $J$. Geodynamics, 41, 358-368

Amalvict, M. and J. Hinderer, 2007, Is the calibration factor stable in time? paper presented at First Asian Workshop on SGs, Taiwan, March 2007.

Boy, J.-P. and J. Hinderer, 2006, Study of the seasonal gravity signal in superconducting gravimeter data, J. Geodyn., 41, 227-233.

Crossley, D., Xu, H., and Van Dam, T., 1998. Comprehensive analysis of 2 years of data from Table Mountain, Colorado, Proc. $13^{\text {th }}$ Int. Symp. Earth Tides, 659-668., eds. Ducarme, B., and Paquet, P., Brussels.

Crossley, D. J. et al., 1999. Network of superconducting gravimeters benefits several disciplines, EOS, 80, pp. 121-126.

Crossley D., Hinderer J., Boy J.-P., de Linage C., 2006, Status of the GGP Staellite project, Bull. d'Inf. Marees Terr, 142, 11423-11432

Crossley, D. and J. Hinderer, 2007, Report of GGP activities to Commission 3, completing 10 years for the worldwide network of superconducting gravimeters, submitted to IAG Proceedings of the IUGG Meeting, Perugia 2007.

de Linage C., Hinderer J., Boy J.-P., 2006, A search on the gravity/height ratio induced by surface loading: theoretical investigation and numerical applications, Bull. d'Inf. Marees Terr, 142, 11451-11460.

Dijksma, R., G.H. Ros, B. de Jong, and P.A. Troch, 2006? Local water storage changes affect in-situ gravity observations at Westerbork, the Netherlands, submitted to PEPI.

Francis, O. and T. van Dam, 2002, Evaluation of the precision of using absolute gravimeters to calibrate superconducting gravimeters, Metrologia, 39, 485-488.

Francis, O., T. van Dam, M. Amalvict, M. de Andrade Sousa, M. Bilker, R. Billson, G. D’Agostino, S. Desogus, R. Falk, A. Germak, O. Gitlein, D. Jonhson, F. Klopping, J. Kostelecky, B. Luck, J. Mäkinen, D. McLaughlin, E. Nunez, C. Origlia, V. Palinkas, P. Richard, E. Rodriguez, D. Ruess, D. Schmerge, S. Thies, L. Timmen, 
M. Van Camp, D. van Westrum and H. Wilmes, 2005, Results of the international ccomparison of absolute gravimeters in Walferdange (Luxembourg) of November 2003, in : IAG Symposia, Gravity, Geoid, and Space Missions, 129, 272-275, Springer, Berlin.

Fukuda, Y., Higashi, T., Keiko Yamamoto, K., and Shuzo Takemoto, S, 2007, A Strategy for detecting temporal gravity variation using AG, SG and space gravimetry, paper presented at First Asian Workshop on SGs, Taiwan, March 2007.

Hinderer, J. and D. Crossley, 2004. Scientific achievements from the first period (1997-2003) of the Global Geodynamics Project using a worldwide network of superconducting gravimeters, J. Geodynamics. 38, $237-262$.

Imanishi, Y., Higashi, T., and Fukuda, Y., 2002. Calibration of the superconducting gravimeter T011 by parallel observation with the absolute gravimeter FG5 210 - a Bayesian approach, Geophys. J. Int., 151, 867-878.

Kroner, C. and T. Jahr, 2006, Hydrological experiments around the superconducting gravimeter at Moxa Observatory, J. Geodyn. 41, 268-275.

Longuevergne, L., G. Ferhat, P. Ulrich, J.-P. Boy, N. Florsch, and J. Hinderer, 2007, Towards physical modeling of local-scale hydrological contribution of soils for precise gravimetric corrections in Strasbourg, paper presented at First Asian Workshop on SGs, Taiwan, March 2007.

Meurers, B., M. van Camp, and T. Petermans, 2007, Correcting superconducting gravity time-series using rainfall modeling at the Vinna and Membach stations and application to Earth tide analysis, J. Geodesy, doi : 10.1007/s00190-007-137-1.

Milly, C., \& Shmakin, A., 2002. Global modeling of land water and energy balances. Part I: the land dynamics (LaD) model, J. of Hydrometeorology, 3, 283-299.

Naujoks, M., C. Kroner, T. Jahr, and A. Weise, 2006, From a disturbing influence to a desired signal: hydrological effects in gravity observations, Bull. d'Inf. Marees Terr. 142, 11359-11360 (Abstract only)

Naujoks, M., C. Kroner, T. Jahr, P. Krause, and A. Weise, 2007. Gravimetric 3D modelling and observation of timedependent gravity variations to improve small-scale hydrological modeling, poster, session HW2004, IUGG XXIV General Assembly, Perugia, Italy.

Okubo, S., S. Yoshida, T. Sato, Y. Tamura, and Y. Imanishi, 1997, Verifying the precision of a new generation absolute gravimeter FG5 - comparison with superconducting gravimeters and detection of oceanic loading tide, Geophys. Res. Lett., 24, (4), 489-492.

Plag, H.-P. and Pearlmanm M., 2007 , The Global Geodetic Observing System: meeting the requirements of a global society on a changing planet in 2020; available on GGOS website, but not for direct citation.

Plag, H.-P., Kreemer, C., \& Hammond, W., 2007. Combination of GPS-observed vertical motion with absolute gravity changes constrain the tie between reference frame origin and Earth center of mass, in: Report of the Seventh SNARF Workshop, held in Monterrey, California, 28 March, 2007 (also Geophys. Res. Lett., submitted).

Rodell, M., Houser, P.R., Jambor, U., Gottschalck, J., Mitchell, K., Meng, C.-J., Arsenault, K., Cosgrove, B., Radakovich, J., Bosilovich, M., Entin, J.K., Walker, J.P., Lohmann, D., and Toll, D., 2004. The global land data assimilation system, Bull. Am. Meteor. Soc. 85 (3), 381-394.

Rogister, Y., M. Amalvict, J. Hinderer, B. Luck, and A. Memin (2007), Absolute gravity measurements in Antarctica during the International Polar Year: in Antarctica: A Keystone in a Changing World-Online Proceedings of the 10th ISAES, edited by A.K. Cooper and C.R. Raymond et al., USGS Open-File Report 2007-1047, Extended Abstract 173, 4 p.

Smith, A. B., J. P. Walker, A. W. Western, K. M. Ellett, R. B. Grayson, and M. Rodell, 2007, Factors affecting the detection of a soil moisture signal in field relative gravity measurements, EOS, Transactions American Geophysical Union, Western Pacific Meeting Supplement, Abstract H23C-02.

Timmen, L., J. Gitlein, J. Muller, H. Denker, J. Makinen, M. Bilker, B.R. Pettersen, O.C.D. Omang, , J.G.G. Svendsen. H. Wilmes, R. Falk, A. Reinhold, W. Hoppe, H.-G. Scherneck, B. Engen, B. G. Harsson, A. Engfeldt, M. Lilje, G. Strykowski, and R. Forsberg, 2006, Observing Fennoscandian gravity change by absolute gravimetry, in: Geodetic Deformation Monitoring: From Geophysical to Engineering Roles, IAG Sympoisia, 131, 193-199.

Van Camp, M., S. D. P. Williams, and O. Francis (2005), Uncertainty of absolute gravity measurements, J. Geophys. Res., 110, B05406, doi:10.1029/2004JB003497.

Wahr, J. H. DaZhong, and A. Trupin, 1995, Predictions of vertical uplift caused by changing polar ice volumes on a vscoelastic earth, Geophys. Res. Lett., 22, 977-980.

Wu, X., M.B. Heflin, E.R. Ivins, and I. Fukumori, 2006, Seasonal and interannual global surface mass variations from multisatellite geodetic data, J. Geophys. Res., 111, B09401, doi:10.1029/2005JB004100.

Wilmes, H., H. Wziontek, R. Falk and P. Wolf, 2007, Investigations of gravity variations and instrumental properties by combing superconducting and absolute gravity measurements, paper presented at First Asian Workshop on SGs, Taiwan, March 2007.

Wziontek, H., R. Falk, H. Wilmes, P. Wolf, 2006, Rigorous combination of superconducting and absolute gravity measurements with respect to instrumental properties, Bull. d'Inf. Marees Terr, 142, 11417-11422 